\title{
Synthesis of substituted 1,2,3-triazoles via $N$-sulfonylaziridines
}

\author{
Victor I. Markov* and Evgenij V. Polyakov \\ *Ukrainian State Chemical-Technological University \\ E-mail:markov@email.dp.ua
}

(received 27 Oct 05; accepted 28 Jan 06; published on the web 28 Feb 06)

\begin{abstract}
Nucleophilic ring-opening of $N$-sulfonylaziridines by sodium azide leads to $\beta$-azidoamines. The regioselectivity of this reaction and new efficient catalyst for such 1,3-dipolar cycloaddition are reported. Several 1,2,3-triazoles have been synthesized and their structures established by X-ray crystallography, MS, IR and ${ }^{1} \mathrm{H}$ NMR spectral data.
\end{abstract}

Keywords: Aziridines, sodium azide, 1,3-dipolar cycloaddition, triazoles

\section{Introduction}

$N$-Substituted aziridines are versatile intermediates for the synthesis of many biologically active compounds that are valuable for new drugs development. There is a growing interest in ring opening reactions of aziridines with various nucleophiles due to their high reactivity and ease of preparation $^{1}$. $\mathrm{N}$-Sulfonyl aziridines are well-known alkylating agents and they are used for the synthesis of several types of heterocyclic compounds ${ }^{2-5}$.

Antibacterial, antifungal, antiviral, anti-inflammatory and analgesic properties of 1,2,3triazoles have been extensively studied. Recently some new 1,2,3-triazole derivatives have been reported as inhibiting tumor proliferation, invasion, and metastasis. ${ }^{6}$

We now report a new method for the synthesis of 1,2,3-triazole derivatives containing aminoethyl groups from $N$-sulfonylaziridines. The process can be carried out stepwise, or as multicomponent ("one-pot") synthesis. Nucleophilic ring-opening of aziridines by sodium azide was studied as a synthetic route to the substituted $\beta$-azidoamines. Regioselective azidolysis of aziridines with $\mathrm{NaN}_{3}$ in the presence of Oxone in aqueous acetonitrile, or $\mathrm{CeCl}_{3}$ was recently reported..$^{2-3}$ However, we have established that azidolysis can be performed without catalyst in DMF in the presence of weak acids, that allowed us to develop a one-pot synthesis of 1,2,3triazoles 3 by the reaction of aziridines 1 with $\mathrm{NaN}_{3}$, acetylenes and catalytic amounts of $\mathrm{Cu}_{2} \mathrm{Cl}_{2}$ in aqueous acetonitrile under reflux for $2-3 \mathrm{~h}$ in good to excellent yields. 


\section{Results and Discussion}

We started this work by studying an aziridine ring-opening with $\mathrm{NaN}_{3}$ in aqueous DMF (DMF: $\mathrm{H}_{2} \mathrm{O}-10: 1$ ) at room temperature. The reaction (Scheme 1) appeared to be $\mathrm{pH}$ dependent. To obtain desired product an addition of acid precursor was necessary, however strongly acidic conditions caused formation of hydrazoic acid, lowering yield of the product 3 .

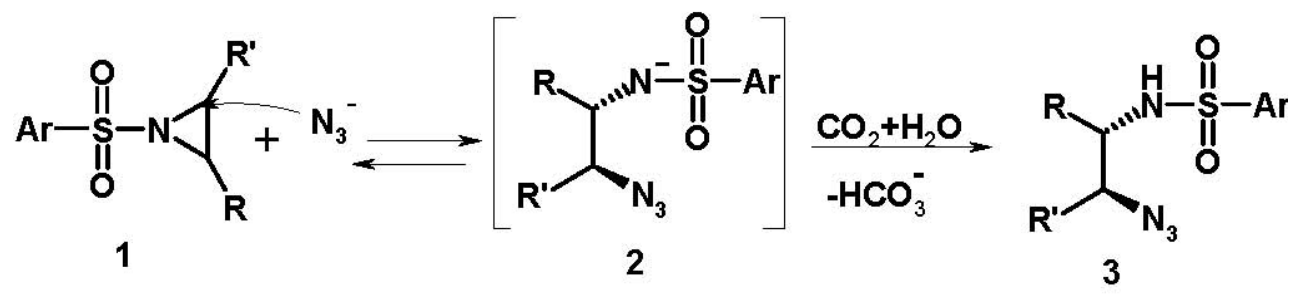

\section{Scheme 1}

It was found that bubbling carbon dioxide through the reaction mixture kept $\mathrm{pH}$ at the optimum level. Reaction was completed when the $\mathrm{pH}$ did not change during several minutes. The acidity of the system $\left(\mathrm{CO}_{2}+\mathrm{H}_{2} \mathrm{O}\right)$ was enough to form anion 2, but not for the generation of $\mathrm{HN}_{3}$ from sodium azide $\left(\mathrm{K}_{\alpha} \mathrm{HN}_{3}=2,0^{*} 10^{-5}, \mathrm{~K}_{\alpha} \mathrm{CO}_{2}+\mathrm{H}_{2} \mathrm{O}=4,45^{*} 10^{-7}\right)$. Precipitation of $\mathrm{NaHCO}_{3}$ was observed during the reaction.

The ring-opening of $\mathrm{N}$-arylsulfonylaziridines derived from styrene and indene 1a-c, $\mathbf{1 g}$ occurred in a regioselective manner with preferential attack at the benzylic position. In contrast, alkyl- $N$-arylsulfonylaziridines, gave $\mathbf{1 h}$-i predominantly resulting from terminal attack by azide.

The reaction of cyclohexyl- $N$ - arylsulfonylaziridines 1d-f with $\mathrm{NaN}_{3}$ provided the desired trans-isomer as a single isomer. Relative stereochemistry was established by ${ }^{1} \mathrm{NMR}$. This conclusion is also supported by X-ray analysis (Figure 1).

The $\beta$-azidoamines obtained are white crystalline products or colorless liquids. They may be used as precursors to 1,2,3-triazoles without further purification.

Our next step was to study 1,2,3-triazole formation by cycloaddition of intermediates $\mathbf{3}$ to mono- and di-substituted alkynes. It was found that reaction of alkyl azides $\mathbf{3}$ with phenylacetylene proceeds efficiently under reflux in toluene in the presence of catalytic amounts of $\mathrm{Cu}_{2} \mathrm{Cl}_{2}$ in 15-30 min. Reaction takes $10-20 \mathrm{~h}$ without catalyst. Presumably terminal alkynes form a complex with $\mathrm{Cu}(\mathrm{I})$ and therefore the reactivity of substrates increases. Usually, precipitation of crude 1,2,3-triazoles, contaminated with copper salts was observed. In order to separate 1,2,3-triazole from $\mathrm{Cu}(\mathrm{I}), \mathrm{H}_{2} \mathrm{~S}$ gas was bubbled into the DMF solution. Copper sulfide was removed by filtration. Final product was obtained after subsequent dilution with cold water and filtration.

Regioselectivity of the process was confirmed by X-ray analysis of compound $4 \mathbf{e}$ (Figure 1 and Table 2). 
Table 1. Nucleophilic addition of $\mathrm{NaN}_{3}$ to $N$-arylsulfonylaziridines 1

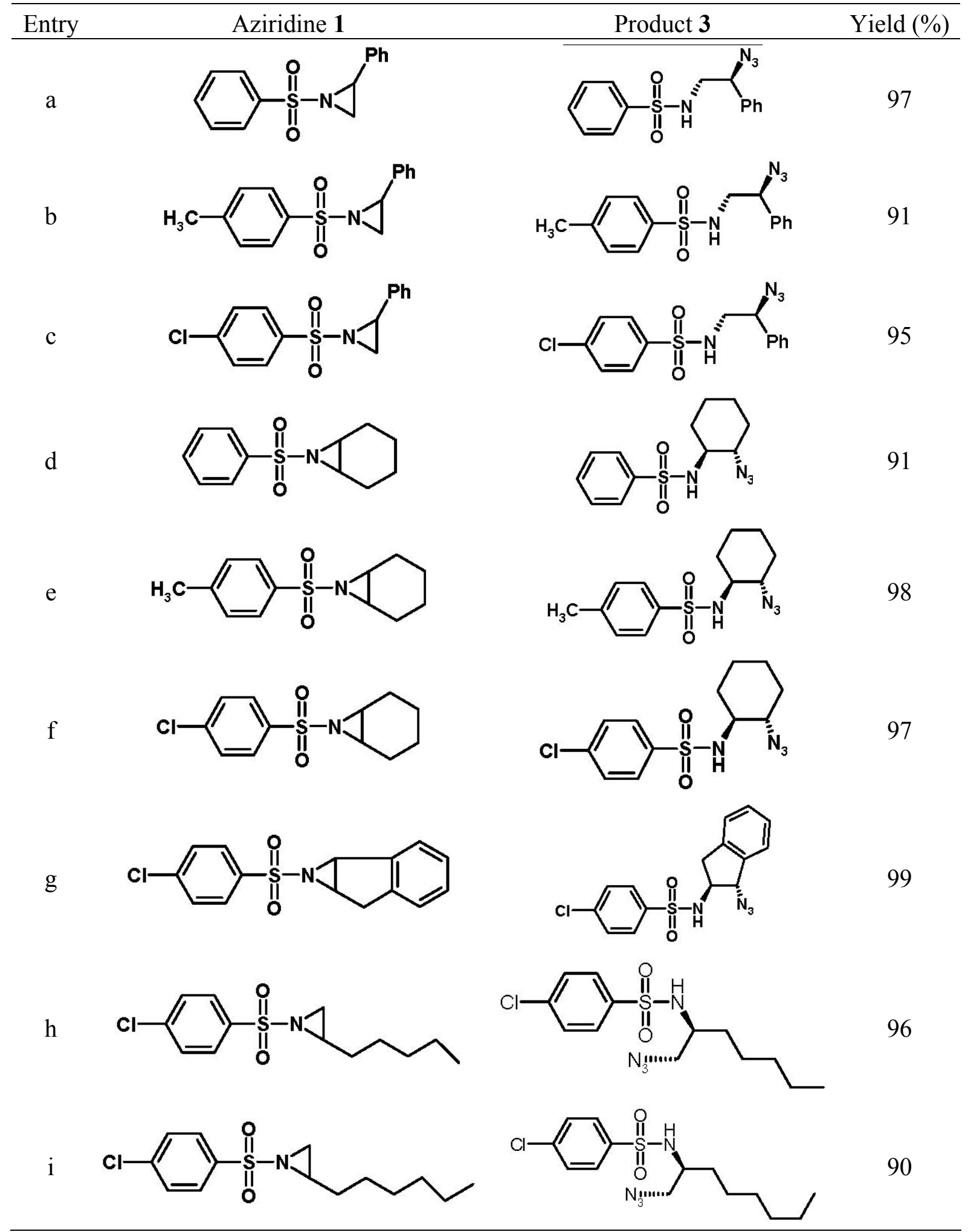




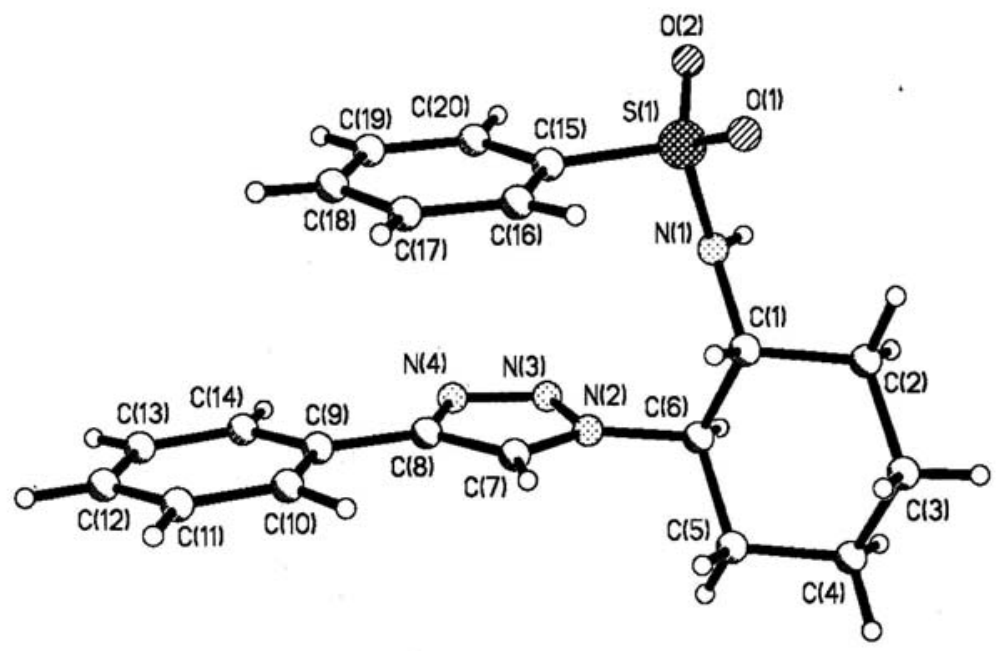

Figure 1

The cyclohexane ring is in the chair conformation (Figure 1). The 4-phenyltriazole substituent is in an equatorial position of the cyclohexane ring (torsion angle $\mathrm{N}(2)-\mathrm{C}(6)-\mathrm{C}(5)-$ $\left.\mathrm{C}(4) 180.0(2)^{\circ}\right)$. The phenyl ring $\mathrm{C}(9) \ldots \mathrm{C}(14)$ and triazole are essentially coplanar (torsion angle $\left.\mathrm{C}(7)-\mathrm{C}(8)-\mathrm{C}(9)-\mathrm{C}(10) 4.4(3)^{\circ}\right)$.

The crystals (Figure 1) form stacks along a crystallographic direction $\left(\begin{array}{lll}0 & 1 & 0\end{array}\right)$ bound by weak intermolecular hydrogen bonds $\mathrm{N}(1)-\mathrm{H}(\mathrm{lN}) \ldots \mathrm{N}(4)^{\prime}(2-\mathrm{x},-\mathrm{y}, 1-\mathrm{z}) \mathrm{H} . . . \mathrm{N} 2.20 \AA$ and N-H...N137.5 ${ }^{\circ}$.

Dimethyl acetylenedicarboxylate also reacted with alkyl azides to produce substituted 1,2,3triazoles. However, in this case catalysis by $\mathrm{Cu}(\mathrm{I})$ was not observed possibly because of the absence of a terminal hydrogen atom in the acetylene, which makes complex formation impossible. Products of type $\mathbf{5}$ were also observed but required a long reaction time to form.
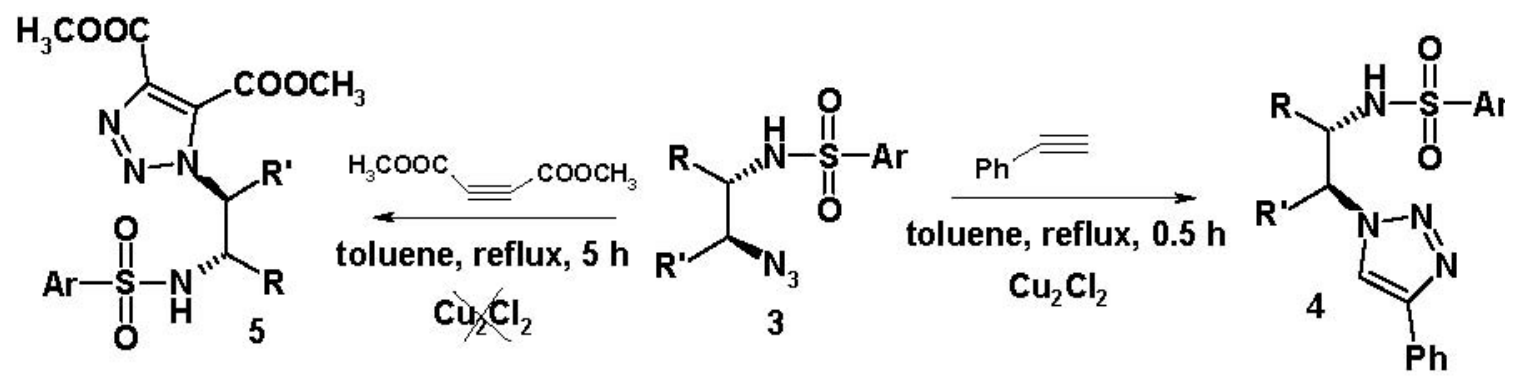

\section{Scheme 2}

We modified the reported two-step sequence, i.e. aziridine ring-opening followed by a cycloaddition, to provide a "one-pot" synthesis of 1,2,3-triazoles 4, 5. This was successfully achieved by using $N$-sulfonylaziridines, $\mathrm{NaN}_{3}$, phenylacetylene and catalytic amounts of $\mathrm{Cu}_{2} \mathrm{Cl}_{2}$ in aqueous acetonitrile (Scheme 3). The advantage of this method is that it produces substantial amounts of 1,2,3-triazoles in a short time, avoiding purification of the intermediated azides. The 
use of a "one-pot" method did not decrease yield of the 1,2,3-triazoles 4 (Table 2).
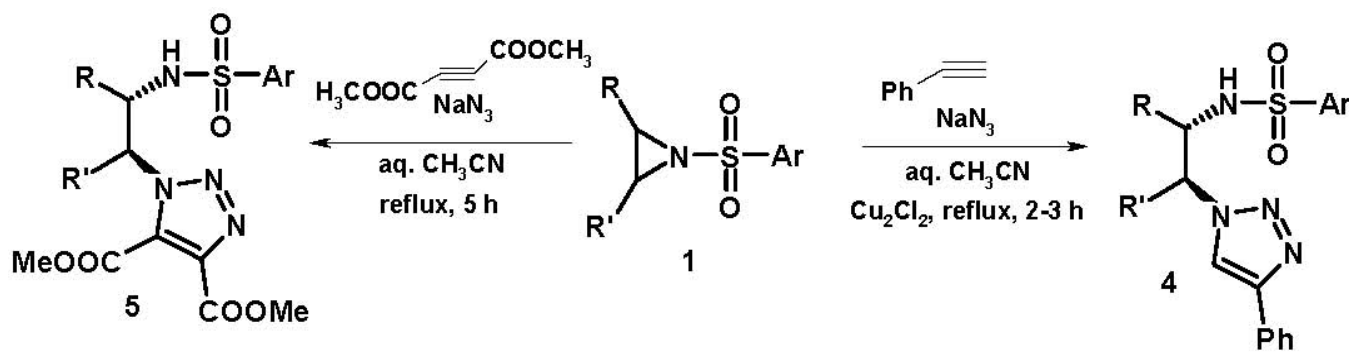

Scheme 3

All 1,2,3-triazole derivatives obtained are white solids with high-melting points. Interestingly, melting points of substituted 1,2,3-triazoles do not dramatically decrease with length of the alkyl chain.

Table 2. 1,3-Dipolar cycloaddition of $\beta$-azidoamines to acetylenes

Entry


Table 2. Continued

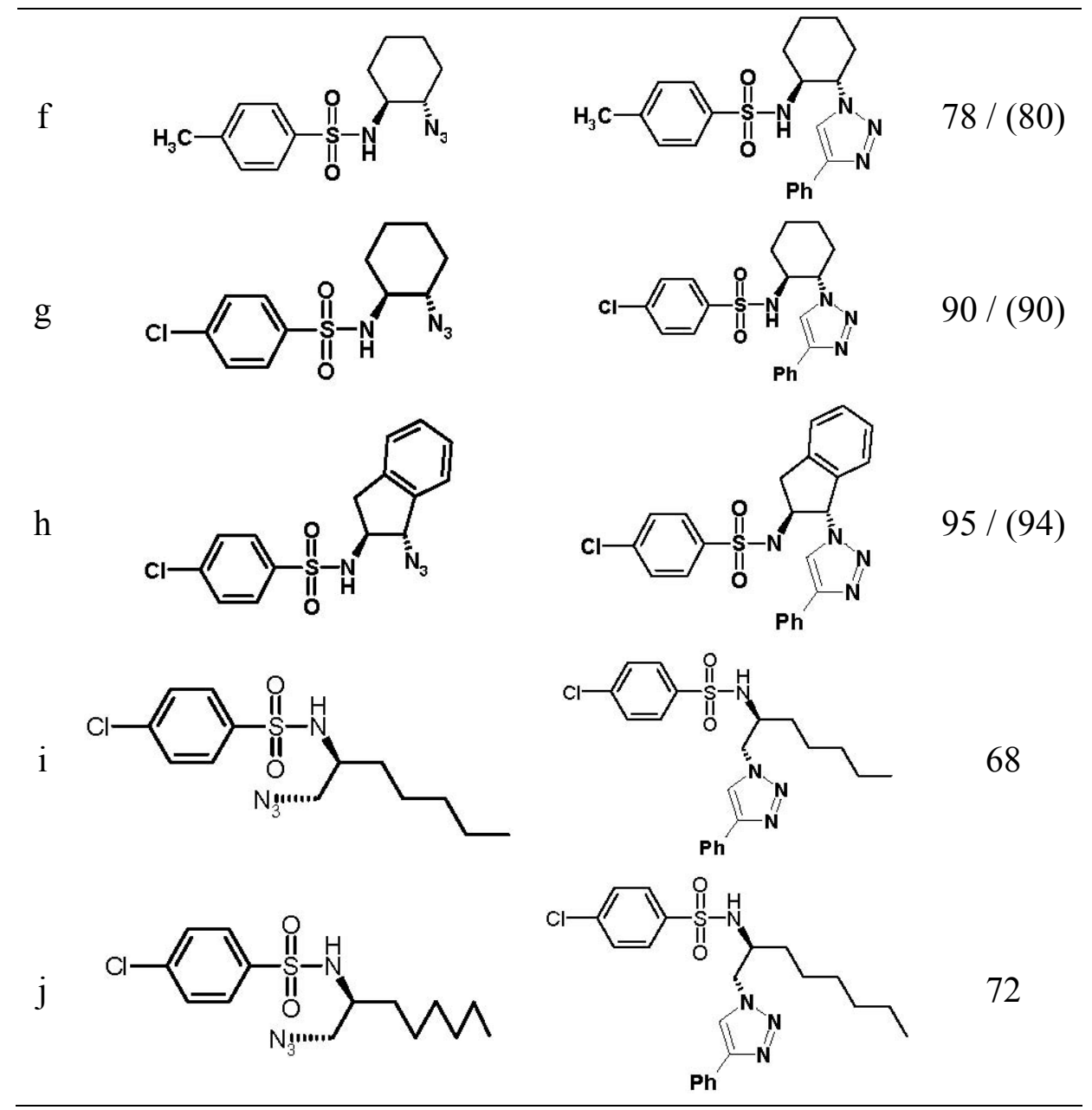

"Yields in parenthesis are given for "one-pot" synthesis of $\mathbf{4}$ and $\mathbf{5}$ from $\mathbf{1}$ as described in the Experimental Section.

\section{Experimental Section}

General Procedures. NMR spectra were recorded on a Bruker DRX200 spectrometer in $\mathrm{CDCl}_{3}$ or DMSO- $\mathrm{d}_{6}$ and chemical shifts $\left(\delta_{\mathrm{H}}\right)$ are reported in parts per million (ppm) relative to tetramethylsilane (TMS). Mass spectra were obtained with a Varian $1200 \mathrm{~L}(-70 \mathrm{eV})$ instrument with straight penetration by an electronic impact, and Infrared spectra were recorded as $\mathrm{KBr}$ pellets or in $\mathrm{CHCl}_{3}$ solution on an Impact 400 ("NICOLET") spectrometer. Melting points were taken in open capillary tubes and are uncorrected.

The single-crystal X-ray study was performed on a Siemens P3/PC diffractometer. Compound 4e, $\left(\mathrm{C}_{20} \mathrm{H}_{22} \mathrm{~N}_{4} \mathrm{O}_{2} \mathrm{~S}\right)\left(20{ }^{\circ} \mathrm{C}\right)$, crystallized in the space group $\mathrm{P} 21_{1} / \mathrm{n}$, monoclinic, from $\mathrm{DMF}$ as colorless rectangular prisms, $a=14.661(5), b=8.641(2), c=15.450(5) \AA, \beta^{\circ}=$ 
104.18(3), $\mathrm{V}=897.8(1) \AA^{3}, \mathrm{M}_{\mathrm{r}}=382.48$.

Lattice constants were determined from least squares refinement on diffractometer angles for 24 automatically centered reflections; $\rho 1.339 \mathrm{Mg} / \mathrm{m}^{3}, \mathrm{Z}=4, \mu=0.194 \mathrm{~mm}^{-1}, \mathrm{~F}(000)=808$. Data collection: monitoring of check reflexion showed no signs of decay. A total of 3748 reflections were measured, (3352 were independent, $\left.\mathrm{R}_{\mathrm{int}}=0.050\right)$.

$\left(\mathrm{F}^{2}: \mathrm{wR}_{2}=0.1046,3337\right.$ reflections $\left(\mathrm{R}_{1}=0.039,2250\right.$ reflections with $\left.\mathrm{F}>4 \sigma(\mathrm{F}), \mathrm{S}=1.002\right)$. All calculations were carried out using the SHELX 97 program package. ${ }^{7-9}$

(For final atom's coordinates see general information)

General procedure of nucleophilic ring opening of $\boldsymbol{N}$-sulfonylaziridines with sodium azide To a solution of $N$-sulfonylaziridine $(0.02 \mathrm{~mol})$ in DMF $(50 \mathrm{~mL})$ was added sodium azide $(0.022 \mathrm{~mol})$ and water $(10 \mathrm{~mL})$. After dissolving the sodium azide, carbon dioxide was bubbled into mixture. The reaction was completed when $\mathrm{pH}$ of the solution became constant. The reaction mixture was diluted with cold water and the product was extracted with ethyl acetate $(3 \mathrm{x}$ $50 \mathrm{~mL}$ ). The extracts were separated, dried over sodium sulfate and solvent removed under vacuum to give (3b-f) either as white crystals or a colorless liquid.

1-(4-Methyl-benzenesulfonamide)-2-phenyl-2-azidoethane (3b). Colorless liquid; IR: 2150, $3275 \mathrm{~cm}^{-1}$; ${ }^{1} \mathrm{H}$ NMR $\left(200 \mathrm{MHz}, \mathrm{CDCl}_{3}\right): \delta=2.45\left(\mathrm{~s}, 3 \mathrm{H}, \mathrm{ArCH}_{3}\right), 2.95-3.10(\mathrm{~m}, 1 \mathrm{H}, \mathrm{CHNH})$, 3.10-3.30 (m, 1H, CHNH), 4.55-4.65 (dd, $\left.J=7.7,5.0 \mathrm{~Hz}, 1 \mathrm{H}, \mathrm{CHN}_{3}\right), 5.30-5.50(\mathrm{~m}, 1 \mathrm{H}, \mathrm{NH})$, 7.05-7.40 (m, 7H, ArH), 7.75 (d, $J=8.0 \mathrm{~Hz}, 2 \mathrm{H}, \mathrm{ArH}) . \mathrm{MS}(\mathrm{EI}): m / z=274\left(\mathrm{M}-\mathrm{N}_{3}\right)+$.

1-(4-Chloro-benzenesulfonamide)-2-phenyl-2-azidoethane (3c). White microcrystals, mp 83$85{ }^{\circ} \mathrm{C}$; IR $\left(\mathrm{cm}^{-1}\right) 3050,3028,2133,1320,1150,1028,540 ;{ }^{1} \mathrm{H}$ NMR $\left(200 \mathrm{MHz}, \mathrm{CDCl}_{3}\right): \delta=$ 3.10-3.20 (m, 2H, $\left.\mathrm{CH}_{2} \mathrm{NH}\right), 4.50-4.70$ (t, $J=7.2$, 4.9 Hz, 1H, $\left.\mathrm{CHN}_{3}\right), 5.0-5.1(\mathrm{t}, 1 \mathrm{H}, \mathrm{NH}), 7.05-$ 7.40 (m, 5H, ArH), 7.6 (d, $J=8.0 \mathrm{~Hz}, 2 \mathrm{H}, \operatorname{ArH}), 7.75$ (d, $J=8.0 \mathrm{~Hz}, 2 \mathrm{H}, \operatorname{ArH})$.

1-(4-Methyl-benzenesulfonamide)-2-azido-cyclohexane (3e). Colorless liquid; IR (neat): 2100, $3320 \mathrm{~cm}^{-1} ;{ }^{1} \mathrm{H}$ NMR $\left(400 \mathrm{MHz}, \mathrm{CDCl}_{3}\right): \delta=1.20-1.40(\mathrm{~m}, 4 \mathrm{H}), 1.60-1.75(\mathrm{~m}, 2 \mathrm{H}), 2.00-2.15$ (m, 2H), 2.45 (s, 3H, $\left.\mathrm{ArCH}_{3}\right), 2.85-2.95\left(\mathrm{~m}, 1 \mathrm{H}, \mathrm{CHN}_{3}\right), 3.00-3.10(\mathrm{~m}, 1 \mathrm{H}, \mathrm{CHNH}), 4.80(\mathrm{~d}, J$ $=5.4 \mathrm{~Hz}, 1 \mathrm{H}, \mathrm{NH}), 7.30(\mathrm{~d}, J=8.1 \mathrm{~Hz}, 2 \mathrm{H}, \mathrm{ArH}), 7.80$ (d, $J=8.1 \mathrm{~Hz}, 2 \mathrm{H}, \mathrm{ArH}) . \mathrm{MS}(\mathrm{EI}): m / z=$ $294(\mathrm{M}+)$.

1-(4-Chloro-benzenesulfonamide)-2-azido-cyclohexane (3f). White microcrystals, mp 88-90 ${ }^{\circ} \mathrm{C} ;{ }^{1} \mathrm{H}$ NMR (200 MHz, DMSO-d ${ }_{6}$ ): $\delta=1.0-1.3$ (m, 4H, cyclohexyl), 1.5 (m, 3H, cyclohexyl), 1.9 (m, 1H, cyclohexyl), 2.9-3.05 (m, 1H, cyclohexyl CH-N $)$, 3.1-3.15 (m, 1H, cyclohexyl CH$\mathrm{NH}), 7.9(\mathrm{~d}, J=8.0 \mathrm{~Hz}, 1 \mathrm{H}, \mathrm{NH}), 7.60(\mathrm{~d}, J=8.1 \mathrm{~Hz}, 2 \mathrm{H}, \mathrm{ArH}), 7.75(\mathrm{~d}, J=8.1 \mathrm{~Hz}, 2 \mathrm{H}, \operatorname{ArH})$.

\section{General procedure of synthesis of $1,2,3$-triazoles $(4,5)$}

A. Using $\boldsymbol{\beta}$-azidoamines. To a solution of $\beta$-azidoamines $(0.01 \mathrm{~mol})$ in $40 \mathrm{~mL}$ of toluene was added phenylacetylene $(0.011 \mathrm{~mol})$ and catalytic amount of $\mathrm{Cu}_{2} \mathrm{Cl}_{2}$. The resulting mixture was refluxed for $30 \mathrm{~min}$. Then triazole contaminated with copper sulfide was filtered off and subsequently purified by bubbling of $\mathrm{H}_{2} \mathrm{~S}$ through the solution in DMF. The filtrate was cooled down and the precipitate was filtrated to give pure triazole. 
B. “One-pot" synthesis 1,2,3-triazoles (4) from $N$-sulfonylaziridines (1). $N$-sulfonylaziridine $(10.0 \mathrm{mmol})$, sodium azide $(11.0 \mathrm{mmol})$, phenylacetylene $(11.0 \mathrm{~mol})$ and catalytic amount of $\mathrm{Cu}_{2} \mathrm{Cl}_{2}(0.55 \mathrm{mmol})$ were added to a mixture of water $(2 \mathrm{~mL})$ and acetonitrile $(20 \mathrm{~mL})$. The reaction mixture was refluxed for 2-3 h. A small amount of product precipitated out during the reflux. The reaction mixture was cooled down, the solid was filtered off, and further purification was done according to the procedure A.

C. Synthesis of 1,2,3-triazole (5) from acetylenedicarboxylic acid ester and azide (3). Acetylenedicarboxylic ester $(0.011 \mathrm{~mol})$ was added to a solution of alkyl azide $3(0.01 \mathrm{~mol})$ in 40 $\mathrm{mL}$ of toluene. Reaction mixture was refluxed for $5 \mathrm{~h}$. A small amount of product precipitated during the reflux. The reaction mixture was cooled down, the solid was filtered off and 1,2,3triazole obtained was recrystallized from aqueous DMF.

D. "One-pot" synthesis of 1,2,3-triazoles (5) using acetylenedicarboxylic acid ester. Synthesis was carried out in $\mathrm{H}_{2} \mathrm{O}: \mathrm{MeCN}(1: 10)$. To the solution of $N$-sulfonylaziridine $(0.01$ mol) were added sodium azide $(0.011 \mathrm{~mol})$, acetylenedicarboxylic ester $(0.011 \mathrm{~mol})$ all at once Reaction mixture was refluxed for $5 \mathrm{~h}$. Resulting solution was cooled down, solid was filtered off and obtained 1,2,3-triazoles were recrystallized from aqueous DMF.

$\boldsymbol{N}$-[2-Phenyl-2-(4-phenyl-1H-1,2,3-triazol-1-yl)ethyl] benzenesulfonamide (4a). General procedure A. White powder, mp 190-192 ${ }^{\circ} \mathrm{C} .{ }^{1} \mathrm{H}$ NMR $\left(200 \mathrm{MHz}, \mathrm{DMSO}-\mathrm{d}_{6}\right): \delta=2.6-2.8(\mathrm{~m}$, 2H, $\left.\mathrm{CH}_{2}-\mathrm{NH}\right), 4.5$ (m, 1H, CH-N(Tr)), 7.9 (m, 1H, NH), 8.5 (s, 1H, (CH(Tr)), 7.1-7.8 (m, 15 H, ArH). Anal. Calcd for $\mathrm{C}_{22} \mathrm{H}_{20} \mathrm{~N}_{4} \mathrm{O}_{2} \mathrm{~S}: \mathrm{C}, 65.33 ; \mathrm{H}, 4.98 ; \mathrm{N}, 13.85$. Found: $\mathrm{C}, 65.43 ; \mathrm{H}, 4.94 ; \mathrm{N}$, 13.65 .

Benzenesulfonamide, 4-methyl- $N$-[2-phenyl-2-(4-phenyl-1H-1,2,3-triazol-1-yl)ethyl] (4b). General procedures A. White solid, mp 201-203 ${ }^{\circ} \mathrm{C} .{ }^{1} \mathrm{H}$ NMR $\left(200 \mathrm{MHz}, \mathrm{DMSO}-\mathrm{d}_{6}\right): \delta=2.55$ (s, 3H, $\mathrm{ArCH}_{3}$ ), 3.5-3.6 (m, 1H, CH-NH), 3.8-3.9 (m, 1H, CH-NH), 5.8 (dd, 1H, CH-N(Tr)), 8.1 $(\mathrm{m}, 1 \mathrm{H}, \mathrm{NH}), 9.05\left(\mathrm{~s}, 1 \mathrm{H},(\mathrm{CH}(\mathrm{Tr})), 7.35-7.95(\mathrm{~m}, 14 \mathrm{H}, \mathrm{ArH})\right.$. Anal. Calcd for $\mathrm{C}_{23} \mathrm{H}_{22} \mathrm{~N}_{4} \mathrm{O}_{2} \mathrm{~S}$ : C, 66.01; H, 5.30; N, 13.39. Found: C, 66.18; H, 5.36; N, 13.08 .

Benzenesulfonamide, 4-chloro- $N$-[2-phenyl-2-(4-phenyl-1H-1,2,3-triazol-1-yl)ethyl] (4c). General procedures A, B. White powder, mp 214-216 ${ }^{\circ} \mathrm{C}$. IR $\left(\mathrm{cm}^{-1}\right) 3250,3032,1350,1150$, 1070, 640. ${ }^{1} \mathrm{H}$ NMR (200 MHz, DMSO-d $): \delta=3.5$ (m, 1H, CH-NH), $3.9(\mathrm{~m}, 1 \mathrm{H}, \mathrm{CH}-\mathrm{NH}), 5.8$ (dd, 1H, CH-N(Tr)), $8.2(\mathrm{~m}, 1 \mathrm{H}, \mathrm{NH}), 8.6$ (s, 1H, $(\mathrm{CH}(\mathrm{Tr})), 7.2-7.9$ (m, 14H, ArH). MS (EI): $m / z=438(5)(\mathrm{M}+), 294(9), 235$ (49.2), 175 (22.4), $145(\mathrm{Ph}-\operatorname{Tr}(\mathrm{M}+))$ (6.8), 116 (100) 111 (p-Cl$\mathrm{Ph}(\mathrm{M}+))(37.3), 104$ (42.0). Anal. Calcd for $\mathrm{C}_{22} \mathrm{H}_{19} \mathrm{ClN}_{4} \mathrm{O}_{2} \mathrm{~S}: \mathrm{C}, 60.20 ; \mathrm{H}, 4.36 ; \mathrm{N}, 12.76$. Found: C, 60.29; H, 4.31; N, 12.16 .

1H-1,2,3-Triazole-4,5-dicarboxylic acid, 1-[2-[[(4-chlorophenyl)sulfonyl]amino]-1-phenylethyl]-, dimethyl ester (5d). General procedure C, D. White solid, mp 160-162 ${ }^{\circ} \mathrm{C}$. ${ }^{1} \mathrm{H}$ NMR $\left(200 \mathrm{MHz}, \mathrm{DMSO}-\mathrm{d}_{6}\right): \delta=3.3\left(\mathrm{~s}, 3 \mathrm{H}, \mathrm{OCH}_{3}\right), 3.8\left(\mathrm{~s}, 3 \mathrm{H}, \mathrm{OCH}_{3}\right), 4.1\left(\mathrm{dd}, 2 \mathrm{H}, \mathrm{CH}_{2}-\mathrm{NH}\right), 5.05$ $(\mathrm{m}, 1 \mathrm{H}, \mathrm{CH}-\mathrm{N}(\mathrm{Tr})), 8.8$ (m, 1H, NH), 9.2 (s, 1H, $(\mathrm{CH}(\mathrm{Tr})), 7.9$ (d, J = 8.0 Hz, 1H, NH), 7.70 (d, $J=8.0 \mathrm{~Hz}, 2 \mathrm{H}, \mathrm{ArH}), 7.2-7.5(\mathrm{~m}, 5 \mathrm{H}, \mathrm{ArH})$. Anal. Calcd for $\mathrm{C}_{20} \mathrm{H}_{19} \mathrm{ClN}_{4} \mathrm{O}_{6} \mathrm{~S}: \mathrm{C}, 50.16 ; \mathrm{H}, 4.00$; N, 11.70. Found: C, 50.11; H, 4.14; N, 12.31 . 
Benzenesulfonamide, $\quad \boldsymbol{N}$-[2-(4-phenyl-1H-1,2,3-triazol-1-yl)cyclohexyl] $\quad$ (4e). General procedures A, B. White solid, mp $245-246{ }^{\circ} \mathrm{C} .{ }^{1} \mathrm{H}$ NMR $\left(200 \mathrm{MHz}, \mathrm{DMSO}-\mathrm{d}_{6}\right): \delta=0.9-1.2(\mathrm{~m}$, $3 \mathrm{H}$, cyclohexyl), 1.5-2.1 (m, 5H, cyclohexyl), 3.5 (m, 1H, cyclohexyl CH-NH), 4.3 (dt, 1H, cyclohexyl CH-N(Tr)), $7.85(\mathrm{~m}, 1 \mathrm{H}, \mathrm{NH}), 8.4(\mathrm{~s}, 1 \mathrm{H},(\mathrm{CH}(\mathrm{Tr})), 7.0-7.7(\mathrm{~m}, 10 \mathrm{H}, \mathrm{ArH})$. Anal. Calcd for $\mathrm{C}_{20} \mathrm{H}_{22} \mathrm{~N}_{4} \mathrm{O}_{2} \mathrm{~S}: \mathrm{C}, 62.81 ; \mathrm{H}, 5.80 ; \mathrm{N}, 14.65$. Found: C, 62.88; H, 5.72; N, 15.30.

Benzenesulfonamide, 4-methyl- $N$-[2-(4-phenyl-1H-1,2,3-triazol-1-yl)cyclohexyl] (4f). General procedures A, B. White solid, mp 230-231 ${ }^{\circ} \mathrm{C} .{ }^{1} \mathrm{H}$ NMR $\left(200 \mathrm{MHz}, \mathrm{DMSO}-\mathrm{d}_{6}\right): \delta=$ 1.1-2.0 (m, 8H, cyclohexyl), 2.05 (s, 3H, $\left.\mathrm{ArCH}_{3}\right), 3.45$ (m, 1H, cyclohexyl CH-NH), $4.6(\mathrm{~m}, 1 \mathrm{H}$, cyclohexyl CH-N(Tr)), $8.4(\mathrm{~m}, 1 \mathrm{H}, \mathrm{NH}), 8.6(\mathrm{~s}, 1 \mathrm{H},(\mathrm{CH}(\mathrm{Tr})), 7.2-7.7(\mathrm{~m}, 9 \mathrm{H}, \mathrm{ArH})$. Anal. Calcd for $\mathrm{C}_{21} \mathrm{H}_{24} \mathrm{~N}_{4} \mathrm{O}_{2} \mathrm{~S}$ : C, 63.61; H, 6.10; N, 14.13. Found: C, 63.67; H, 6.21; N, 13.50.

Benzenesulfonamide, 4-chloro- $\mathrm{N}$-[2-(4-phenyl-1H-1,2,3-triazol-1-yl)cyclohexyl] (4g). General procedures A, B. White solid, mp 250-251 ${ }^{\circ} \mathrm{C} .{ }^{1} \mathrm{H}$ NMR $\left(200 \mathrm{MHz}, \mathrm{DMSO}-\mathrm{d}_{6}\right): \delta=$ 1.1-1.45 (m, 3H, cyclohexyl), 1.5-2.0 (m, 5H, cyclohexyl), 3.65 (m, 1H, cyclohexyl CH-NH), $4.4(\mathrm{dt}, 1 \mathrm{H}$, cyclohexyl CH-N(Tr)), $8.1(\mathrm{~d}, J=8.0 \mathrm{~Hz}, 1 \mathrm{H}, \mathrm{NH}), 8.45(\mathrm{~s}, 1 \mathrm{H},(\mathrm{CH}(\mathrm{Tr}), 7.70$ (d, $J$ $=8.0 \mathrm{~Hz}, 2 \mathrm{H}, \mathrm{ArH}), 7.5(\mathrm{~d}, J=8.0 \mathrm{~Hz}, 2 \mathrm{H}, \mathrm{ArH}), 7.2-7.5(\mathrm{~m}, 5 \mathrm{H}, \mathrm{ArH}) . \mathrm{MS}(\mathrm{EI}): m / z=416$ $(\mathrm{M}+)$ (8.1), 272 (29.5), 213 (76.2), 175 (47.2), $145(\mathrm{Ph}-\mathrm{Tr}(\mathrm{M}+))$ (7.8), $111(p-\mathrm{Cl}-\mathrm{Ph}(\mathrm{M}+))(79.0)$, 81(cyclohexene (M+·)) (100). Anal. Calcd for $\mathrm{C}_{20} \mathrm{H}_{21} \mathrm{ClN}_{4} \mathrm{O}_{2} \mathrm{~S}$ : C, 57.62; H, 5.08; N, 13.44 . Found: C, 57.34; H, 5.00; N, 13.47 .

Benzenesulfonamide, 4-chloro- $N$-[2,3-dihydro-1-(4-phenyl-1 H-1,2,3-triazol-1-yl)-1H-inden2-yl] (4h). General procedures A, B. White solid, mp 295-297 ${ }^{\circ} \mathrm{C} .{ }^{1} \mathrm{H}$ NMR (200 MHz, DMSO$\left.\mathrm{d}_{6}\right): \delta=2.5-3.0\left(\mathrm{~m}, 2 \mathrm{H},-\mathrm{CH}_{2}-\right), 4.5(\mathrm{~s}, 1 \mathrm{H}, \mathrm{CH}-\mathrm{N}(\mathrm{Tr})), 6.0(\mathrm{~m}, 1 \mathrm{H}, \mathrm{CH}-\mathrm{NH}), 8.8(\mathrm{~s}, 1 \mathrm{H}, \mathrm{NH})$, 8.5 (s, $1 \mathrm{H},(\mathrm{CH}(\mathrm{Tr})), 6.70-8.0(\mathrm{~m}, 13 \mathrm{H}, \mathrm{ArH}) . \mathrm{MS}(\mathrm{EI}): \mathrm{m} / z=307\left({ }^{37} \mathrm{Cl}, \mathrm{M}+\right)(1.6), 305\left({ }^{35} \mathrm{Cl}\right.$, $\mathrm{M}+),(12.5), 279$ (1.3), 212 (1.2), 178 (2.2), $111(p-\mathrm{Cl}-\mathrm{Ph}(\mathrm{M}+))(26.2), 103$ (100). Anal. Calcd for $\mathrm{C}_{23} \mathrm{H}_{19} \mathrm{ClN}_{4} \mathrm{O}_{2} \mathrm{~S}: \mathrm{C}, 61.26 ; \mathrm{H}, 4.25 ; \mathrm{N}, 12.42$. Found: $\mathrm{C}, 61.01 ; \mathrm{H}, 4.21 ; \mathrm{N}, 12.30$.

Benzenesulfonamide, 4-chloro- $N$-[1-[(4-phenyl-1H-1,2,3-triazol-1-yl)methyl]hexyl] (4i). Phenylacetylene $(0.011 \mathrm{~mol})$ and catalytic amount of $\mathrm{Cu}_{2} \mathrm{Cl}_{2}$ were added to the solution of $\beta$ azidoamine $(0.01 \mathrm{~mol})$ in $40 \mathrm{~mL}$ of toluene. Resulting mixture was refluxed for $30 \mathrm{~min}$. Precipitation of yellow crude 1,2,3-triazole were observed. After that, the mixture was filtered, and filtrate was cooled. Precipitate was filtered and filtrate was extra diluted with hexane. Obtained crude 1,2,3-triazole was purified by recrystallization from DMF. White solid, mp 165$167{ }^{\circ} \mathrm{C} ;{ }^{1} \mathrm{H}$ NMR $\left(200 \mathrm{MHz}, \mathrm{DMSO}-\mathrm{d}_{6}\right): \delta=0.8-0.9(\mathrm{~m}, 3 \mathrm{H}), 1.0-1.15(\mathrm{~m}, 6 \mathrm{H}), 1.5-1.4(\mathrm{~m}$, 2H), 3.7 (dd, $1 \mathrm{H}, \mathrm{CH}-\mathrm{N}(\mathrm{Tr})), 4.3-4.45\left(\mathrm{~m}, 2 \mathrm{H}, \mathrm{CH}_{2} \mathrm{NH}\right), 7.9$ (d, J=8.0 Hz, $\left.1 \mathrm{H}, \mathrm{NH}\right), 8.3(\mathrm{~s}, 1 \mathrm{H}$, $(\mathrm{CH}(\mathrm{Tr})), 7.70(\mathrm{~d}, J=8.0 \mathrm{~Hz}, 2 \mathrm{H}, \mathrm{ArH}), 7.5(\mathrm{~d}, J=8.0 \mathrm{~Hz}, 2 \mathrm{H}, \mathrm{ArH}), 7.1-7.5$ (m, 5H, ArH). MS (EI): $m / z=432(\mathrm{M}+)$ (12.5), 274 (7.2), 230 (8.3), 229(58.1), $145(\mathrm{Ph}-\mathrm{Tr}(\mathrm{M}+))(5.2), 111(p-\mathrm{Cl}-$ $\mathrm{Ph}(\mathrm{M}+))(100)$. Anal. Calcd for $\mathrm{C}_{21} \mathrm{H}_{25} \mathrm{ClN}_{4} \mathrm{O}_{2} \mathrm{~S}: \mathrm{C}, 58.26 ; \mathrm{H}, 5.82 ; \mathrm{N}, 12.94$. Found: C, 57.99; H, 5.94; N, 12.90.

Benzenesulfonamide, 4-chloro- $N$-[1-[(4-phenyl-1H-1,2,3-triazol-1-yl)methyl]heptyl] (4j). Obtained according to 4 i. White solid, mp $165-167{ }^{\circ} \mathrm{C} ;{ }^{1} \mathrm{H}$ NMR $\left(200 \mathrm{MHz}, \mathrm{DMSO}-\mathrm{d}_{6}\right): \delta=$ 0.6-0.7 (m, 3H), 0.85-1.05 (m, 8H), 1.5-1.4 (m, 2H), $3.5(\mathrm{dd}, 1 \mathrm{H}, \mathrm{CH}-\mathrm{N}(\mathrm{Tr})), 4.25-4.4(\mathrm{~m}, 2 \mathrm{H}$, $\left.\mathrm{CH}_{2} \mathrm{NH}\right), 8.0(\mathrm{~d}, J=8.0 \mathrm{~Hz}, 1 \mathrm{H}, \mathrm{NH}), 8.4(\mathrm{~s}, 1 \mathrm{H},(\mathrm{CH}(\mathrm{Tr})), 7.70(\mathrm{~d}, J=8.0 \mathrm{~Hz}, 2 \mathrm{H}, \mathrm{ArH}), 7.5$ 
(d, $J=8.0 \mathrm{~Hz}, 2 \mathrm{H}, \mathrm{ArH}), 7.2-7.5(\mathrm{~m}, 5 \mathrm{H}, \mathrm{ArH}) . \mathrm{MS}(\mathrm{EI}): \mathrm{m} / z=446(\mathrm{M}+)(16.6 \%), 302(7.5 \%)$, 290 (3.2\%), 243(100\%), $145(\mathrm{Ph}-\operatorname{Tr}(\mathrm{M}+))(8.3 \%), 111(p-\mathrm{Cl}-\mathrm{Ph}(\mathrm{M}+))(89 \%)$. Anal. Calcd for $\mathrm{C}_{22} \mathrm{H}_{27} \mathrm{ClN}_{4} \mathrm{O}_{2} \mathrm{~S}: \mathrm{C}, 59.11 ; \mathrm{H}, 6.09 ; \mathrm{N}, 12.53$. Found: C, 58.82; H, 6.11; N, 12.14 .

\section{$\underline{\text { Supplementary Material is Available }}$}

\section{Acknowledgments}

The authors thank Scientific and Technological Corporation "Institute for Single Crystals" (Dr. O. Shishkin, Dr. V. Chebanov, Dr. E. Gladkov, Dr. R. Zubatuk) for their support in characterization of obtained compounds.

Financial support by the state-grant $0103 \mathrm{U} 001186$.

\section{References}

1. Markov, V. I.; Burmistrov, S. I.; Danilei` ko, D. A.; Doroshenko, V. A.; Gella, I. M. Rus. J. Org. Chem. (Russ. Ed.) 1971, 7, 602; Chem. Abstr. 1971, 75, 5588.

2. Hu, X. E. Tetrahedron 2004, 60, 2701.

3. Sabitha, G.; Babu, R. S.; Rajkumar, M.; Yadav, J. S. Synthesis 2002, 15, 2254.

4. Sabitha, G.; Babu, R. S.; Rajkumar, M.; Yadav, J. S. Org. Lett. 2002, 4, 343.

5. Abu-Orabi, S. T. Molecules 2002, 7, 302.

6. Heng, S. D.; Kun, W. I.; Qing, L. W., Bin, Q., Zhang, Z. Y. J. Chinese Chem. Soc. 2000, 47, 343.

7. Zefirov, N. S.; Palyulin, V. A.; Dashevskaya, E. E. J. Phys. Org. Chem. (Russ. Ed). 1990, 3, 147.

8. Zefirov, Y. V.; Zorkiy, P. M. Russ. Chem. Rev. (Russ. Ed). 1989, 5, 713.

9. Sheldrick, G.M. SHELX97. PC Version. A system of computer programs for the crystal structure solution and refinement. Rev.2, 1998. 